\title{
Age-Dependent Effects of ALK5 Inhibition and Mechanism of Neuroprotection in Neonatal Hypoxic-Ischemic Brain Injury
}

\author{
Brian H. Kim ${ }^{\text {a }}$ Mariano Guardia Clausi ${ }^{a} \quad$ Michelle J. Frondelli ${ }^{a} \quad$ Israel C. Nnah ${ }^{b}$ \\ Chaitali Saqcena $^{b}$ Radek Dobrowolski $^{\text {b Steven W. Levison }}{ }^{a}$ \\ a Department of Pharmacology, Physiology, and Neuroscience, Rutgers New Jersey Medical School, and \\ ${ }^{b}$ Federated Department of Biological Sciences, Rutgers University/New Jersey Institute of Technology, \\ Newark, NJ, USA
}

\section{Keywords}

Cell death · Premature birth · Birth asphyxia .

Neuroprotection · Encephalopathy · Autophagy

\begin{abstract}
Neonatal encephalopathy due to hypoxic-ischemic (HI) brain injury triggers a wave of neuroinflammatory events attributed to causing the progressive degeneration and functional deficits seen weeks after the initial insult. In a recent set of studies, we evaluated the therapeutic efficacy of a small molecule antagonist for ALK5 (activin-like kinase 5 ), TGF- $\beta$ receptor in a rat model of moderate perinatal $\mathrm{HI}$ and found significant improvements in neurologic outcomes. Here, we have extended those studies to evaluate the efficacy of delayed TGF- $\beta$ receptor antagonism on postnatal day (P) 6 and $\mathrm{P} 9 \mathrm{HI}$ rat pups with and without hypothermia. The ALK5 receptor antagonist SB505124 was administered systemically by osmotic pump beginning 3 days following $\mathrm{HI}$. Extending our earlier data set that showed protection of the hippocampus in P6 pups treated with SB505124, these animals sustained less damage to their hippocampi and had improved performance on the Morris water maze (MWM) when tested on $\mathrm{P} 60$ versus vehicle-treated $\mathrm{HI}$ animals. By contrast, SB505124 did not improve sensorimotor
\end{abstract}

\section{KARGER}

() 2017 S. Karger AG, Basel

E-Mail karger@karger.com

www.karger.com/dne deficits and exacerbated hippocampal and thalamic volume loss when administered 3 days after $\mathrm{HI}$ to P9 pups. SB505124-treated rats injured on P9 tended to perform worse than their vehicle-treated counterparts on MWM, and SB505124 treatment did not preserve hippocampal or thalamic neurons in P9 pups when combined with hypothermia. To elucidate the mechanism whereby ALK5 inhibition reduced neuronal death in the P6 $\mathrm{HI}$ model, we assessed levels of autophagy markers in neurons of the neocortex, hippocampus, and thalamus, and in the subcortical white matter, and found that SB505124 increased numbers of autophagosomes and levels of lipidated LC3 (light chain 3), a key protein known to mediate autophagy. Altogether, our results demonstrate that there is a dynamic switch in the CNS response to TGF- $\beta_{1}$ that occurs around P9 in rats where TGF- $\beta$ signaling inhibition worsens functional outcomes. This response is similar to the outcome of antagonizing TGF- $\beta$ signaling in adult stroke and other CNS disease models. We conclude that attenuating TGF- $\beta_{1}$ signaling will likely be an effective treatment for HI-related encephalopathy in moderately preterm infants, offering protection of the neocortex, hippocampus, and thalamus with enhanced cerebral autophagy contributing to the decrease in the extent of progressive neuronal cell death.

(c) 2017 S. Karger AG, Basel

Steven W. Levison, PhD

Department of Neurology and Neuroscience, Rutgers New Jersey Medical School 205 South Orange Avenue

Newark, NJ 07103 (USA)

E-Mail levisosw@ rutgers.edu 


\section{Introduction}

Hypoxic-ischemic (HI) brain injury is the predominant contributor to neonatal encephalopathy arising from inadequate oxygenation/perfusion to the fetus or from asphyxiating complications during birth [1]. This injury represents a major cause of neurological morbidity in infants, producing permanent cognitive, motor, and sensory deficits. Therapeutic hypothermia is approved for the treatment of moderate neonatal encephalopathy and has been shown to improve survival if administered within the first $6 \mathrm{~h}$ of life $[2,3]$. However, the therapy offers limited functional recovery and comes with its own set of complications. Hypothermia is contraindicated in preterm infants and is not fully neuroprotective, thus preterm infants too often face lifelong learning disabilities and impairments [4-6]. Most recently, the therapy has been linked to bowel dismotility and intestinal perforations, in addition to coagulopathy, arrhythmia, and increased rates of infection [7, 8]. Consequently, therapies are desired that provide synergistic effects when combined with hypothermia or that are effective when used alone to restore the course of brain development.

Our laboratory has established that the cytokine transforming growth factor (TGF)- $\beta_{1}$ is subacutely produced in cerebral HI, which is a highly significant finding given that this cytokine is typically produced during the resolution phase of inflammation that classically occurs 2 weeks after the insult [9]. In the CNS, TGF- $\beta_{1}$ has been shown to modulate microgliosis and astrogliosis [10]. Most recently, we showed that inhibiting the type I TGF- $\beta$ receptor (ALK5 or activin-like kinase 5) using the small molecule SB505124 significantly improved neurological outcome, even when administered as late as 3 days after injury in a rat model of perinatal HI [11]. Given the efficacy of ALK5 inhibition in preterm injury, we sought to validate this therapeutic approach for use in a nearterm infant model as a standalone drug and as combinatorial therapy with hypothermia.

Here, we subjected rats on P6 to the Vannucci HI model of brain injury to study late preterm injury and we subjected rats on $\mathrm{P} 9$ to $\mathrm{HI}$ to model near-term injury. We investigated the efficacy of ALK5 inhibition alone in the P6 rats and evaluated efficacy of ALK5 inhibition both alone and together with therapeutic hypothermia in P9 rats.

\section{Materials and Methods}

\section{Rodents}

All experiments were performed in accordance with research guidelines set forth by Institutional Animal Care and Use Committee of Rutgers New Jersey Medical School and were in accordance with the National Institute of Health Guide for the Care and Use of Laboratory Animals (NIH publication No. 80-23) revised in 1996 and the ARRIVE guidelines. Time pregnant Wistar rats on embryonic day 18 of gestation were purchased from Charles River Laboratories (Wilmington, MA, USA). Following delivery, litter sizes were adjusted to 12 pups per litter, and efforts were made to ensure the number of each sex and pup weights were equal and consistent. Animals were group housed and kept on a 12-h light:dark cycle with ad libitum access to food and autoclaved water. Rat pups remained with the dam until the day of HI injury.

\section{Neonatal HI Brain Injury}

Cerebral $\mathrm{HI}$ in 6-day-old rat pups (P6, day of birth = P0; mean body mass $=15 \mathrm{~g}$ ), as a model of late preterm injury, was performed as previously described $[9,11]$. Briefly, rats were anesthetized with isoflurane (3-4\% induction, $1-2 \%$ maintenance) prior to right common carotid artery cauterization. A special effort was made to carefully isolate the carotid without damaging other structures contained within the carotid sheath (i.e., internal jugular vein and vagus nerve). The neck incision was sutured with 4-0 surgical silk. Following a 1-h recovery period, rats were exposed to $75 \mathrm{~min}$ of hypoxia in humidified $8 \%$ oxygen/nitrogen balance. Likewise, P9 pups were subjected to $65-75$ min of hypoxia to achieve a similar level of moderate injury. Sham rats were anesthetized and underwent isolation of the right common carotid without cauterization to mimic similar insult to HI-injured animals but without the ischemic event. Rats were randomly assigned to experimental groups after HI injury. Sample sizes per experiment were chosen to achieve sufficient statistical power with minimal numbers of animals based on pilot studies.

\section{Therapeutic Hypothermia}

Immediately following hypoxia, rats were randomly designated for hypothermia treatment or normothermia during recovery. Therapeutic hypothermia was performed based on a validated model as reported previously $[12,13]$. Hypothermic animals were cooled in a glass chamber placed within a $29^{\circ} \mathrm{C}$ water bath to achieve a rectal temperature of $32^{\circ} \mathrm{C}$, determined by rectal probe (Physitemp, Clifton, NJ, USA). Animals in the normothermia group were placed in a $34.5^{\circ} \mathrm{C}$ water bath to achieve a rectal temperature of $36^{\circ} \mathrm{C}$. Animals in both treatment groups were exposed to room air during the duration of treatment. Following treatment, pups from both groups were returned to their home cages.

\section{SB505124 Drug Delivery}

Three days following HI injury, rats were anesthetized (isoflurane, $3-4 \%$ induction, $1-2 \%$ maintenance), and an incision was made in the subcapsular region. Osmotic pumps (Alzet 1007D; Durect, Cupertino, CA, USA) were loaded with either vehicle (sodium citrate buffer with $30 \%$ DMSO v/v) or ALK5 pharmacological inhibitor, 2-(5-benzo[1, 3] dioxol-5-yl-2-tert-butyl-3H-imidazol-4-yl)-6-methylpyridine hydrochloride hydrate (SB505124) (Sigma-Aldrich; St. Louis, MO, USA) and implanted subdermally. The incision was then sutured with 4-0 surgical silk, and animals

Dev Neurosci 2017;39:338-351

DOI: $10.1159 / 000477490$
339
ALK5 Inhibition in Hypoxic-Ischemic

Brain Injury 
were surveyed twice per day for signs of infection and/or distress. The 1007D model osmotic pump can continuously deliver solutions for 7 days and was left implanted until animal euthanization, or it was removed 10 days following implantation. Animals subjected to behavioral testing and volumetric analyses were treated with SB505124 for 7 days, following the same dosing schedule as in our previous study [11]. Animals used for Western blot and immunofluorescence analyses were treated with SB505124 for 4 days.

\section{Behavioral Testing}

Sensorimotor Testing

Three weeks following HI injury, rats were subjected to a comprehensive battery of sensorimotor function tests aggregated into a modified neurological severity score ( $\mathrm{mNSS}$ ) as described in detail previously [11]. Tests were conducted by an investigator blinded to the experimental groups.

\section{Morris Water Maze}

Morris water maze (MWM) trials were conducted in a $72 \times 15$ inch pool. The pool was filled with water to a depth of 10 inch with room temperature water. The escape cylinder platform measured 5 inch in diameter with a height of $9.5 \mathrm{inch}$. We ensured pool placement remained consistent in the testing space, and cues (i.e., colored construction paper shape cutouts) were placed at the north, west, and east walls of the room prior to testing. Testing began between 9 and 10 a.m. every morning for a period of 6 consecutive days. Animals were left in the testing room approximately $1 \mathrm{~h}$ before the start of trials every morning to allow the rats to acclimate to their environment. An investigator blinded to the experimental groups conducted the trials.

Rats were subject to pretraining for the first day of examination. For the pretraining, the escape platform was placed in the center of the pool with the top of the platform above the surface of the water. The pool water remained clear for this session to ensure visibility of the platform. Each rat was placed on the platform for $10 \mathrm{~s}$ to allow the animal to orient to the apparatus and to the cues. The rat was then removed from the platform and placed tail-end first into a far-end of 1 of 3 test quadrants facing the wall of the pool. The rat was allowed to swim for $30 \mathrm{~s}$ before being removed from the water to be returned tail-end first onto the platform. The rat was then allowed to re-orient for a period of $20 \mathrm{~s}$ before being placed back into pool at a different test quadrant than the first. The trial was then repeated 2 additional times so that each rat visited each test quadrant once. After 3 consecutive trials, rats were removed from the apparatus, dried with paper towel under a heating lamp, and returned to their home cages.

On days $2-5$, the water was colored with nontoxic black tempera paint to occlude visibility of the platform and to provide a contrast to the rats for accurate software tracking. The escape platform was placed in the southwest quadrant of the pool, and the water level was raised such that the top of the platform was submerged approximately 1 inch below the surface. Each rat was once again placed tail end into one of test quadrants, defined as a quadrant in which the escape platform was not located (i.e., NW, NE, and SE quadrants). ANY-maze (Stoelting Co., Wood Dale, IL, USA) tracking began recording the trial as soon as the rat was placed into the pool with a maximum swim time set to $65 \mathrm{~s}$. If the rat failed to reach the platform in the allotted time, it was guided to the platform. Once the platform was reached, each rat was given $10 \mathrm{~s}$ to orient before being removed from the apparatus. Each rat was tested twice in each test quadrant for a total of 6 trials per day. Rats were dried after each trial under a heating lamp and checked for dryness at the end of trials each day. On the final day of examination (day 6), a probe test was administered as a measure of spatial memory. The platform was removed from the target quadrant, and each rat was placed in the NE quadrant and allowed to swim freely for $60 \mathrm{~s}$. Upon ending the trial, the rat was removed and dried to be returned to the home cage. In the P6 HI injury group, all trials from each session were analyzed using the ANY-maze software while latency to the platform was measured manually in the P9 group.

\section{Western Blot Analyses}

One week after HI injury, microdissected brain tissue of the affected cortex, striatum, and corpus callosum from the injured (ipsilateral) and uninjured (contralateral) hemispheres was collected either as a single wedge of infarcted tissue or as microdissected regions. The tissue was homogenized and then sonicated in lysis buffer; $30 \mu \mathrm{g}$ of denature protein was loaded into a $4-12 \%$ Bis-Tris gel (Invitrogen, Carlsbad, CA, USA), and $5 \mu \mathrm{L}$ of Amersham ECL Rainbow Marker was loaded as a molecular weight standard (GE Life Sciences, Pittsburgh, PA, USA). Proteins were transferred onto nitrocellulose and incubated with primary antibody $(\mathrm{Ab})$ : LC3 (light chain 3; rabbit polyclonal Ab; Cell Signaling, 1:1,000), SQSTM1/p62 (guinea pig polyclonal Ab; Progen 1:1,000), $\beta$-tubulin (mouse monoclonal Ab; Santa Cruz, 1:1,000). Membranes probed for LC3 and $\beta$-tubulin were washed with $0.01 \%$ TBS-Triton $\mathrm{X}-100$, incubated in HRP-conjugated secondary $\mathrm{Ab}$, and washed, and bands were visualized using Western Lightning chemiluminescence reagent (PerkinElmer, Wellesley, MA, USA). Membranes probed for $\mathrm{p} 62$ were likewise washed with $0.01 \%$ TBS-Triton X-100 and incubated in IRDye 680LT secondary Ab (LI-COR; Lincoln, NE, USA). Imaging for LC3 and $\beta$-tubulin was performed using a BioRad ChemiDoc Imaging System combined with Image Lab software (Hercules, CA, USA). Imaging for p62 was performed using a LI-COR Odyssey Imaging System. Quantification was performed using ImageJ software.

\section{Brain Histology and Immunofluorescence}

One week after HI injury, rats were deeply anesthetized with sodium pentobarbital before intracardiac perfusion with $4 \%$ paraformaldehyde (PFA) in PBS. Brains were postfixed overnight in $4 \%$ PFA/PBS, cryoprotected with $30 \%$ sucrose overnight, and embedded in Tissue-Tek OCT matrix (Sakura Finetek, Torrance, CA, USA). Serial coronal sections $25-30 \mu \mathrm{m}$ thick were taken through the hippocampal and thalamic regions using a cryostat at $-14^{\circ} \mathrm{C}$ and mounted on slides. Sections were then incubated with primary antibodies in $1 \%$ goat serum $/ 0.05 \%$ Triton $\mathrm{X}-100 / \mathrm{PBS}$ at $4^{\circ} \mathrm{C}$ overnight. Primary antibodies included: (1) p62 (guinea pig polyclonal Ab; Progen, 1:100); (2) LC3 (rabbit polyclonal Ab; Cell Signaling, 1:100); (3) NeuN (mouse monoclonal Ab; Millipore, $1: 100)$. Sections were washed with $0.05 \%$ Triton X-100/PBS 3 times for $30 \mathrm{~min}$ and incubated with secondary antibodies for $2 \mathrm{~h}$ at room temperature. Secondary antibodies included: (1) donkey anti-guinea pig Cy5; (2) donkey anti-rabbit Alexa 488; (3) donkey anti-mouse Cy3; (3) (all from Jackson ImmunoResearch; 1:250). Sections were washed with $1 \%$ goat serum $/ 0.05 \%$ Triton X-100/ PBS 3 times and mounted in Prolong Gold Antifade Mountant with DAPI (ThermoFisher Scientific, Waltham, MA, USA). Confocal images were collected by an investigator blinded to each 
group using a Zeiss spinning-disk microscope and ZEN software. All acquired images used the same acquisition and laser settings, set initially using sham (uninjured) samples. Images were processed such that p62 signals (detected in the far-red region) were converted to red, and NeuN signals (detected in the red region) were converted to blue for signal colocalization studies. Signal intensities were quantified using ImageJ with plugins as described by Hamilton [14] in 2009.

\section{LC3-p62 Colocalization Analyses}

Three images were captured from each brain ( $n=3$ animals/ group) for a total of 45-60 cells/determination in each brain region (cortex, white matter, hippocampus, and thalamus). The images were coded to blind the investigator to group identities, and Autothreshold (provided in Fiji plugin bundle for ImageJ) was performed to eliminate potential bias during elimination of background signals. Manders' colocalization coefficient (MCC) was determined using the JACoP plugin for ImageJ. The M1 value reported represents the fractional overlap of the p62 signal in compartments containing the LC3 signal. A value of 1 represents complete overlap of both signals; a value of zero represents no overlap. The benefits of reporting the MCC over the Pearson colocalization coefficient or Manders' overlap coefficient is reviewed by Dunn et al. [15] in 2011.

Volumetric Analysis

Nine weeks after HI injury, rats were deeply anesthetized with sodium pentobarbital before intracardiac perfusion with $4 \% \mathrm{PFA} /$ PBS. Whole brains were extracted and dehydrated in $70 \%$ ethanol and then embedded in paraffin. Ventricular size was assessed from sections taken $0.5 \mathrm{~mm}$ from the bregma while hippocampal and thalamic volumes were assessed from sections $-3 \mathrm{~mm}$ from the bregma. All sections were cresyl violet stained and imaged using an Olympus BX51 microscope (Center Valley, PA, USA) and captured by a Q-imaging Retiga-2000R CCD camera (Surrey, BC, Canada). Volumes of the hippocampus and thalamus were determined using the Cavalieri principle associated with the counting point method as described elsewhere $[16,17]$.

\section{Data Analysis and Statistics}

Raw data from image analyses and behavioral tests were imported into Prism (Graphpad Software; La Jolla, CA, USA) for statistical analyses using ANOVA followed by the Tukey post hoc intergroup comparison. Results from MWM testing were analyzed by ANOVA followed by the Bonferroni method. Graphs were produced in Prism, and error bars denote standard error of means. In the water maze data set, comparisons were made to the sham group $(*)$ or the vehicle group (\#) and represented as: ${ }^{* / \#} p<0.05$, ${ }^{* * / \# \#} p<0.01,{ }^{* * * / \# \# \#} p<0.001$. Significance among colocalization coefficients in immunofluorescence imaging was determined using ANOVA followed by the Tukey post hoc intergroup comparison.

\section{Results}

Delayed SB505124 Administration Improves Spatial

Learning and Memory after HI on P6

In an earlier study, we showed that volumes of ipsilateralhippocampiwere significantlypreserved in SB505124- treated HI pups compared to vehicle-treated HI pups when the injury was induced on P6 [11]. To determine whether the observed structural preservation translated to retained hippocampal function and whether the neuroprotection was long lasting, we tested $\mathrm{HI}$ injured rats in the MWM task at 2 months of age to assess spatial learning and memory (Fig. 1a). As expected, vehicle-treated rats required more time to reach the platform than SB505124-treated $\left({ }^{\#} p<0.05\right)$ and sham rats $(* * p<0.001)$, with significantly increased latencies into the third day of training. We found no significant difference in latencies in SB505124-treated rats compared to sham rats by ANOVA followed by post hoc Bonferroni analysis (Fig. 1b; ANOVA; main effect of treatment: $\mathrm{F}_{(2,285)}=2.176$; main effect of day: $\mathrm{F}_{(3,285)}=11.07, p<0.0001$; interaction: $\left.\mathrm{F}_{(6,285)}=2.222, p<0.05\right)$. To further assess water maze performance, we calculated path efficiencies for each trial, defined as the straight line distance between the entry point of the rat and the platform divided by the total distance traveled by the rat upon pool entry - a value of 1 indicates perfect efficiency while values $<1$ indicate decreasing efficiency. Vehicle-treated rats were significantly less efficient at swimming to the platform than SB505124-treated $\left(^{\# \# \#} p<0.001\right)$ and sham $(* * * p<0.001)$ rats into the third and fourth days of training (Fig. 1c; ANOVA; main effect of treatment $\mathrm{F}_{(2,286)}=7.335, p<$ 0.001 ; main effect of day: $\mathrm{F}_{(3,286)}=9.327, p<0.0001$; interaction: $\left.F_{(6,286)}=1.679\right)$. To determine if swimming speed accounted for differences in latencies, we measured mean swim velocities. Surprisingly, vehicle-treated rats swam significantly faster across all 4 days of training, reaching a velocity of $0.287 \pm 0.017 \mathrm{~m} / \mathrm{s}$ on training day 2 and remaining elevated until day 3. In contrast, both SB505124-treated and sham rats reduced swimming speed by day 3 (Fig. 1d; ANOVA; main effect of treatment: $\mathrm{F}_{(2,385)}=22.64, p<0.0001$; main effect of day: $\mathrm{F}_{(3,385)}=3.236, p<0.05$; interaction: $\left.\mathrm{F}_{(6,385)}=0.9014\right)$. Vehicle-treated rats also traveled longer distances to reach the platform per trial across the first 3 days of training (Fig. 1e; ANOVA; main effect of treatment: $\mathrm{F}_{(2,385)}=$ $14.79, p<0.0001$; main effect of day: $\mathrm{F}_{(3,385)}=19.23, p<$ 0.0001 ; interaction: $\left.F_{(6,385)}=1.374\right)$. During the probe trial, we found no differences in the preference for the target (platform) quadrant for all 3 groups (Fig. 1f).

\section{Delayed SB505124 Treatment Does Not Improve} Sensorimotor Recovery and Exacerbates Degeneration in the P9 Model of Neonatal HI

In our previous studies, we observed preservation of both sensorimotor function and axons of the corticospi-
ALK5 Inhibition in Hypoxic-Ischemic Brain Injury
Dev Neurosci 2017;39:338-351 DOI: $10.1159 / 000477490$ 


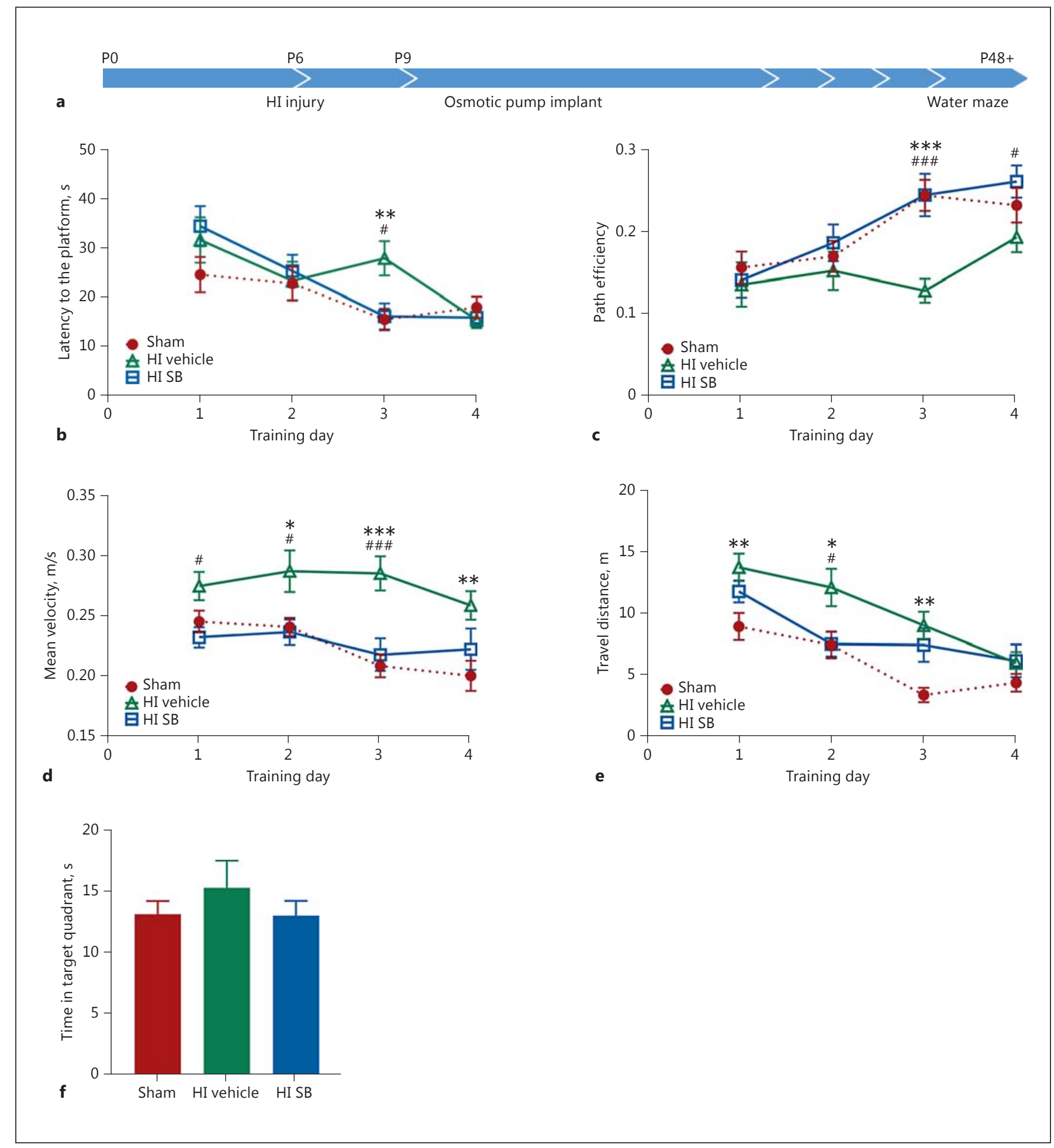

Fig. 1. SB505124 improves spatial learning and memory functions after hypoxic-ischemic (HI) injury on P6. a Rats were evaluated 6 weeks after HI injury on P6 using the Morris water maze (MWM) to assess hippocampal function. For each day of training, data were averaged across 6 trials per animal. b Latency to find the platform across training days for MWM. c Path efficiency measures across training days. d Swimming rates across all 4 days of training. e Distances traveled per trial. $\mathbf{f}$ Total time spent in the target quadrant during the probe trial. $n=6$ /group; means $\pm \mathrm{SEM} ;{ }^{*} p<0.05$, ${ }^{* *} p<0.001,{ }^{* * *} p<0.0001$, vehicle-treated vs. sham rats; ${ }^{\#} p<0.05$, \#\# $p<0.001$, \#\# $p<0.0001$, vehicle-treated vs. SB505124-treated rats (ANOVA followed by the Bonferroni post hoc test). 


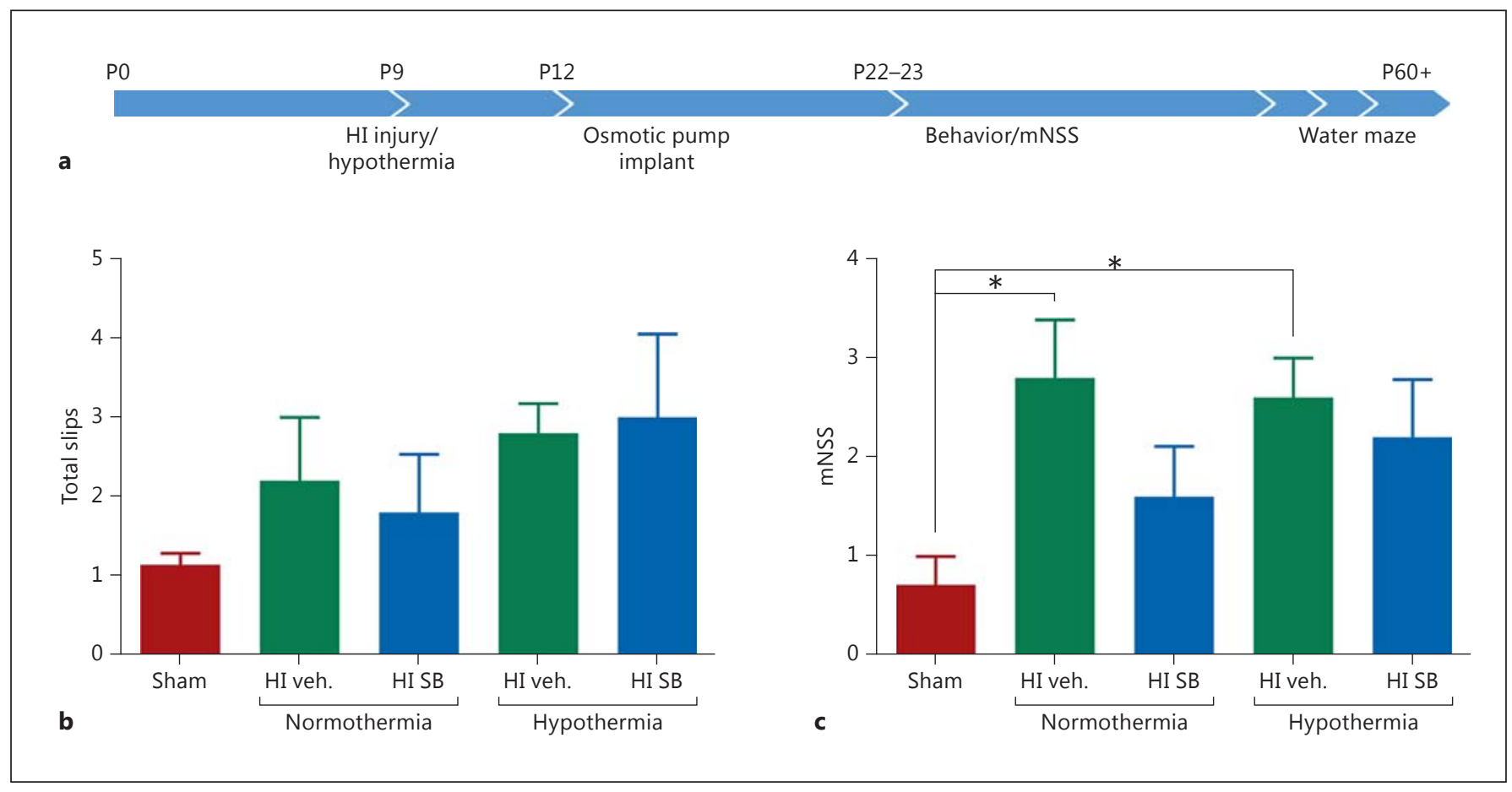

Fig. 2. SB505124 has no effect on sensorimotor deficits after hypoxic-ischemic (HI) injury on $\mathrm{P} 9$. Three weeks after $\mathrm{HI}$ injury on $\mathrm{P} 9$, behavioral tests were performed to assess sensorimotor function. a Outline of the experimental paradigm of HI injury and behavioral testing. Osmotic pumps deliver loaded drug solution over the course of 10 days. Following HI injury, animals were divided into 2 groups to either receive $4 \mathrm{~h}$ of hypothermia or remain at room temperature (normothermia). Sensorimotor testing began 13 days following $\mathrm{HI}$ injury in which rats were given a pretraining day $24 \mathrm{~h}$ before the start of testing. $\mathbf{b}$ Results of the beam walking tests. c Results of the modified neurological severity score (mNSS) which evaluates 9 tasks graded on a scale from 0 to 9 (normal score, 0 ; maximal deficit scored, 9). $n=5-7$ rats/group; means \pm SEM; $* p<0.05$ (ANOVA followed by the Tukey post hoc test). nal tract in P6 HI pups treated systemically with SB505124 [11]. Impressed with the efficacy of this small molecule receptor antagonist, we hypothesized that SB505124 would be beneficial for older rat pups. Moreover, as SB505124 protected the hippocampus and thalamus, both brain regions that are not protected by therapeutic hypothermia, it was of interest to establish whether SB505124 would provide additional neuroprotection to these structures beyond that achieved with hypothermia alone. To test this hypothesis, we performed HI on P9injured rat pups and performed behavioral and morphometric analyses to assess efficacy of neuroprotection (Fig. 2a). Rats were assessed on the beam walking test where they were trained on a $2.5-\mathrm{cm}$-width beam $24 \mathrm{~h}$ prior to being tested using a narrower (2-cm-width) beam. HI rats tended to have more foot slips than sham animals in both normothermia and the HI pups treated with hypothermia, but there was no significant benefit of SB505124 treatment either alone or in conjunction with hypothermia (Fig. 2b). When rats were scored using the
mNSS for motor function, tactility, and proprioception, HI-injured rats performed significantly worse on tasks than sham rats $(* p<0.05)$, and despite the trend to some improvement with SB505124, this was not statistically significant (Fig. 2c). In this moderate injury model, rats treated with therapeutic hypothermia did not perform significantly differently better than their normothermic counterparts, and the treatment with SB505124 did not significantly improve their sensorimotor function (Fig. $2 b, c)$.

Upon completing the behavioral analyses, the brains from vehicle- and SB505124-treated animals were evaluated histologically for the extent of damage and degeneration (Fig. 3). We focused our analyses on the hippocampus and thalamus, as these structures were significantly preserved in SB505124-treated rats following injury on P6 [11]. Contrary to the data obtained from studies on the younger pups, hippocampal volumes were significantly reduced in SB505124-treated animals compared to vehicle-treated rats regardless of hypothermia 


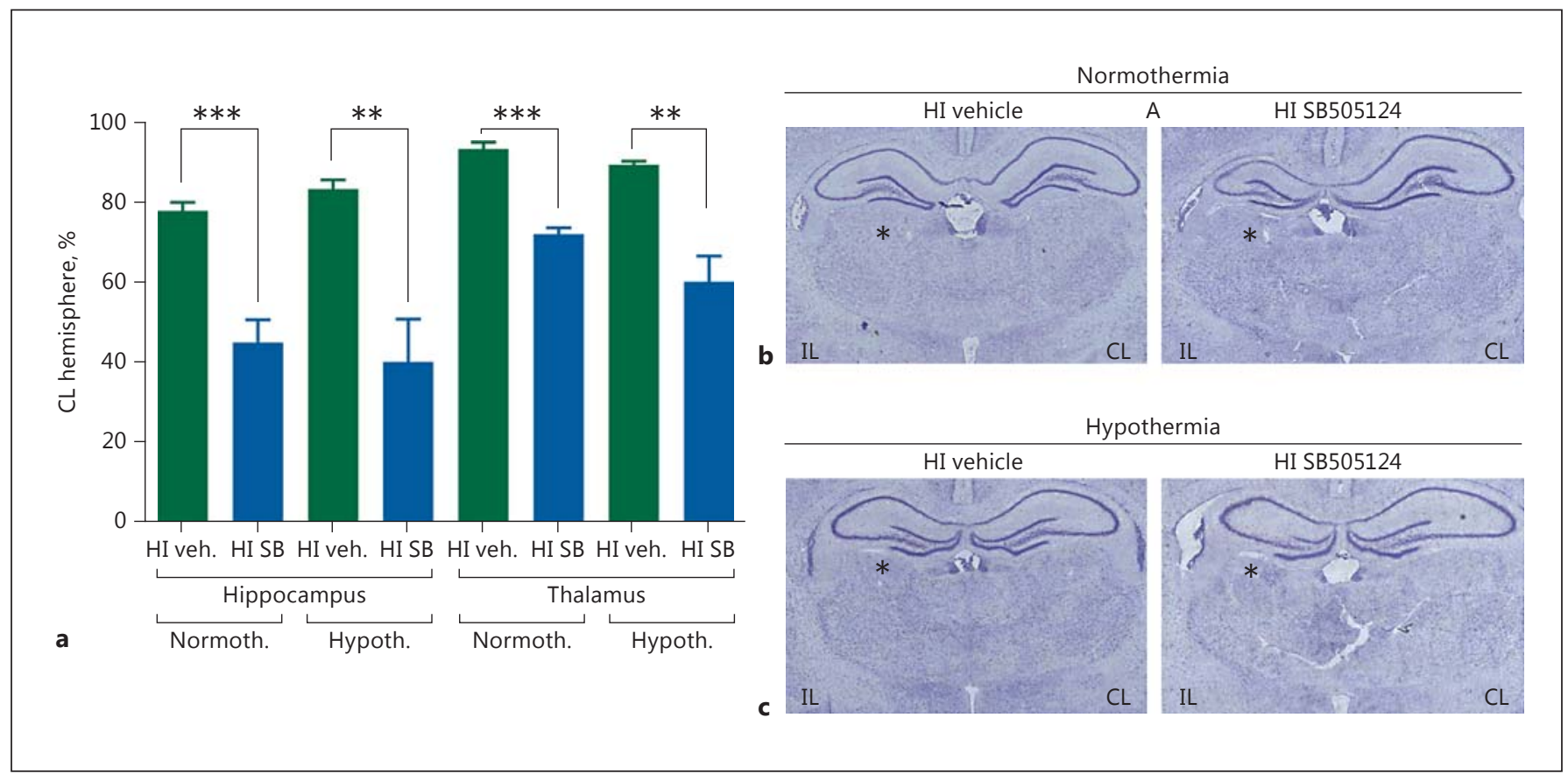

Fig. 3. Systemically administered SB505124 exacerbates hippocampal and thalamic volume loss after hypoxic-ischemic (HI) injury on P9. Cresyl violet-stained sections $3 \mathrm{~mm}$ from the bregma were analyzed 10 weeks after HI injury on P9. a Volumes of the hippocampus and thalamus of the injured, ipsilateral (IL) hemi-

$\left.{ }^{* * *} p<0.001\right)$ or normothermic recovery $\left({ }^{* *} p<0.001\right)$. A similar result was obtained from analyses of the thalamus, where there was a significant volume reduction in SB505124-treated rats compared to vehicle-treated controls (region marked by an asterisk in Fig. 3b). There was no significant difference in the ventricular index between vehicle- and SB505124-treated HI animals (data not shown).

Spatial Learning and Memory Does Not Improve with Delayed SB505124 Treatment after HI on P9

To assess whether the exacerbated loss of hippocampal volume translated to comparatively worse behavioral performance, we tested P9 HI-injured rats in the MWM task 6-9 weeks after injury. SB505124-treated HI rats tended to take longer to reach the platform across the 4 training days compared to sham and $\mathrm{HI}$ vehicle-treated rats (Fig. 4a, b). However, there was no significant difference in latencies to reach the platform amongst groups across the 4 days of training in HI injury with normothermic recovery (Fig. 4a; ANOVA; main effect of treatment: $\mathrm{F}_{(2,227)}=1.95$, nonsignificant; main effect of day: $\mathrm{F}_{(3,227)}=5.569, p<0.001$; interaction: $\mathrm{F}_{(6,227)}=0.7362$, sphere were measured and normalized to contralateral (CL) structures. b, c Representative images of volume loss of hippocampal and thalamic regions (marked by an asterisk) compared to the $\mathrm{CL}$ hemisphere. $n=5$ /group; means \pm SEM; ${ }^{* *} p<0.001,{ }^{* * *} p<0.0001$ (Student $t$ test).

nonsignificant). In $\mathrm{HI}$ injury with hypothermia treatment, latencies to reach the platform in vehicle- and SB505124treated rats closely resembled those of sham rats. Hypothermia followed by SB505124 treatment had no significant effect on improving latency to the reach platform (Fig. 4b; ANOVA; main effect of treatment: $\mathrm{F}_{(2,263)}=1.409$, nonsignificant; main effect of day: $\mathrm{F}_{(3,263)}=5.525, p<$ 0.0001 ; interaction: $\mathrm{F}_{(6,263)}=0.1037$, nonsignificant).

\section{Delayed SB505124 Enhances Cerebral Autophagy after HI Injury on P6}

From our data, it was clear that ALK5 inhibition enhanced neuronal survival following HI injury on P6; therefore, we performed studies to establish how this small molecule receptor antagonist was reducing neuronal cell death. Carloni et al. [18] had shown that neuronal autophagy is higher in the neocortex and hippocampus of the ipsilateral hemisphere by $72 \mathrm{~h}$ after $\mathrm{HI}$ in $\mathrm{P} 7$ rats, returning to baseline levels within a period of 5 days. Treatment using 3-methyladenine, an inhibitor of PI3K that effectively terminates autophagy induction, after $\mathrm{HI}$ injury significantly increased cellular necrosis $[18,19]$. Based on this evidence, we hypothesized that SB505124 


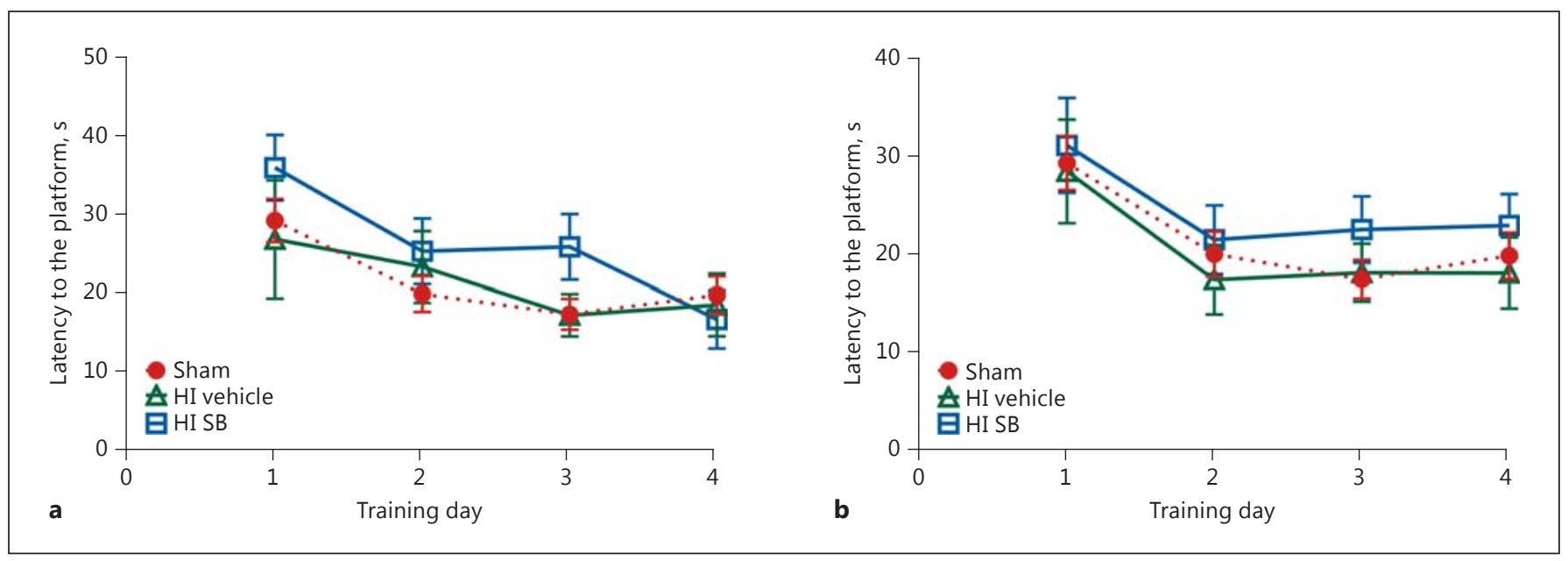

Fig. 4. SB505124 does not improve spatial learning and memory functions after hypoxic-ischemic (HI) injury on P9. Rats were evaluated 6-9 weeks after HI injury on P9 using the Morris water maze (MWM) to assess hippocampal function. a, b Training days for MWM. For each day of training, data were averaged across 6 trials per animal. Latency to reach the platform across 4 training days for HI injury with normothermia (a) and hypothermia treatment (b). $n=5-7 /$ group; means \pm SEM.

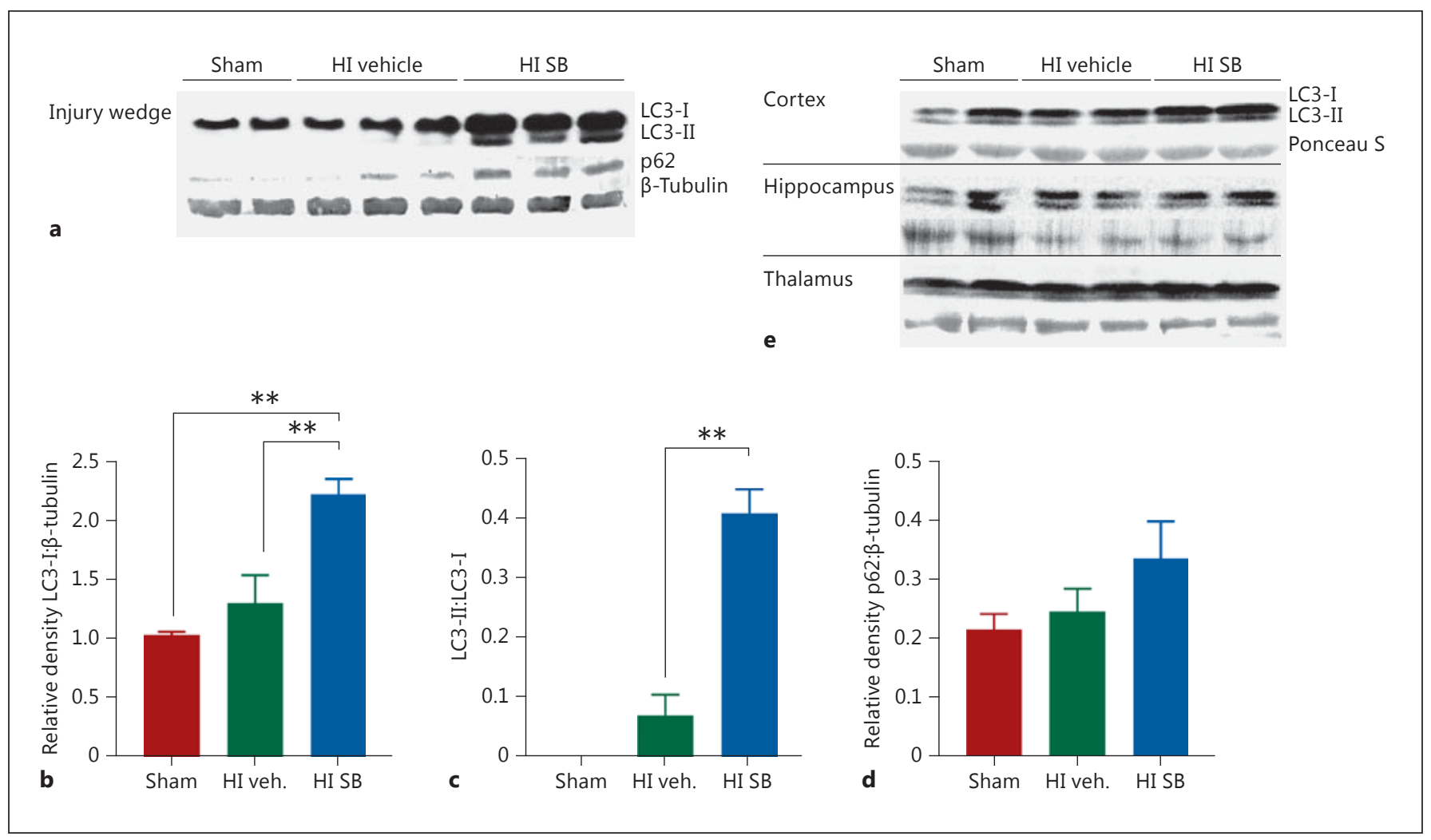

Fig. 5. SB505124 improves autophagy following hypoxic-ischemic (HI) injury on P6. SB505124 or vehicle was administered via osmotic pump for 4 days prior to euthanization and sample preparation for Western blot. a Representative blot for LC3, p62, and $\beta$-tubulin (loading control) from a wedge of the injured hemisphere. b Quantitative analysis of band optical densities for LC3-I. c Ratio of LC3-II:LC3-I band densities. d Optical band densities for p62. $n=3$ /group; means \pm SEM; ${ }^{*} p<0.05,{ }^{* *} p<0.001$ (ANOVA followed by the Tukey post hoc test). e Representative blot for LC3 and Ponceau S (loading control) from an independent experiment where neocortices, hippocampi, and thalami were microdissected and analyzed separately.
ALK5 Inhibition in Hypoxic-Ischemic Brain Injury
Dev Neurosci 2017;39:338-351 DOI: $10.1159 / 000477490$ 
might be inducing autophagy to reduce the extent of neurodegeneration.

Induction of selective autophagy is marked by the lipidation of the microtubule-associated protein LC3 and its association with sequestosome1 (SQSTM1/p62), leading to autophagosome formation containing ubiquitinated substrates [20]. One week following HI injury on P6, SB505124-treated animals showed increased expression of LC3-II in the injured forebrain compared to sham $\left({ }^{*} p<0.05\right)$ and vehicle-treated HI pups $\left({ }^{* *} p<0.001\right)$ as determined by Western blot (Fig. 5a, b). Furthermore, there was a significant increase in the ratio of LC3-II to LC3-I (Fig. 5c). While LC3-II was elevated in SB505124treated rats compared to vehicle-treated rats, there was no significant difference in levels of p62 (Fig. 5d). To determine whether there were regional differences in autophagy, samples of the neocortex, hippocampus, and thalamus were selectively dissected from the injured hemisphere and evaluated again by Western blot. SB505124 treatment increased LC3-II expression in the neocortex, but did not change LC3-II levels in the hippocampus or thalamus (Fig. 5e). Correspondingly, immunofluorescence imaging of the neocortex indicated a significant increase in the expression of LC3 and p62 in neurons $(\mathrm{NeuN}+)$, consistent with active autophagosome formation (Fig. 6a-c). The signal intensity for p62 tended to increase following SB505124 treatment but was not significant in the corpus callosum, hippocampus, or thalamus (Fig. 6b, f, Fig. 7b, f). The signal intensity for LC3 also increased and was statistically significantly in the neocortex, hippocampus, and thalamus but not in the corpus callosum (Fig. 6c, g, Fig. 7c, g). An increase in LC3+ puncta was clearly evident across all regions (Fig. 6a, Fig. 7a). Confocal images of the neocortex, white matter, hippocampus, and thalamus indicated a significant increase in signal colocalization of LC3 and p62 in the neocortex, thalamus, hippocampus, and white matter with SB505124 treatment, as determined by MCC (Fig. 6d, h, Fig. 7d, h). A significant increase in fractional overlap of p62 in LC3+

Fig. 6. SB505124 stimulates the association of autophagic markers LC3 and p62 following hypoxic-ischemic (HI) injury on P6 in the neocortex and subcortical white matter. One week after HI injury on P6, samples of the injured forebrain were analyzed by immunofluorescence. SB505124 or vehicle was administered via osmotic pump beginning 3 days after injury and maintained for 4 days prior to intracardiac perfusion; $30-\mu \mathrm{m}$ sections were stained with anti-p62 (red), anti-LC3 (green), and anti-NeuN (blue) markers. a Representative cortical neurons in the ischemic penumbra of injury with sham cortex as control. e Corpus callosal white matter compartments of neurons ( $\mathrm{NeuN}+)$ and subcortical white matter (NeuN-) in addition to an increased formation of LC3 puncta is consistent with enhanced autophagosomal assembly seen in autophagy.

\section{Discussion}

Prolonged neuroinflammation past the first $24 \mathrm{~h}$ of injury is a major contributor to persistent neuronal cell death and poor behavioral outcomes [1, 21,22]. Thus, there is great interest in establishing which cytokines are responsible for the long-lasting consequences of CNS neuroinflammation. We and others have shown that TGF- $\beta_{1}$ stimulates immature astrocyte proliferation, and that immature astrocytes can produce proinflammatory mediators $[9,11,23,24]$. Furthermore, TGF- $\beta_{1}$ exerts its effects on astrocytes through the ALK5 receptor, and we recently demonstrated that administering an ALK5 antagonist, SB505124, beginning 3 days after HI injury to P6 rat pups attenuated astrogliosis, neuronal cell death, corticospinal axon loss, and ventriculomegaly, and induced CNS levels of IL-6 and IL-1a [11]. Antagonizing the ALK5 receptor also increased subcortical white matter myelination and improved sensorimotor function. In the studies described here, we compared the efficacy of inhibiting the TGF- $\beta$ type 1 receptor after HI injury in the P6, late preterm model and in the $\mathrm{P} 9$ term model of $\mathrm{HI}$ injury.

In the P6 model of late preterm brain injury, SB505124 treatment improved spatial learning and memory function, with SB505124-treated rats performing very similarly to sham rats on successive training days, especially on the latency to find the platform. This result extends our previous data set in which SB505124 treatment rescued hippocampal and thalamic neurons from degenerating [11]. A possible confounding factor for the water maze is that the rats may reach the platform sooner by virtue of swimming speed rather than their ability to

delineated by lack of NeuN+ staining. b, c, f, g Integrated optical densities (IOD) of p62 and LC3 fluorescence of sham, vehicletreated $\mathrm{HI}$, and SB-treated $\mathrm{HI}$ groups in each respective region. Insets depict representative cells enlarged an additional $\times 2$. d, h Manders' colocalization coefficient (M1) for the fractional overlap of p62 signals in compartments containing LC3 signals for the cortex and white matter. $n=3-5$; means \pm SEM; ${ }^{*} p<0.05$, ${ }^{* *} p<$ $0.001,{ }^{* * *} p<0.0001$ (ANOVA followed by the Tukey post hoc test). Scale bars in merged images, $10 \mu \mathrm{m}$.

(For figure see next page.)
346

Dev Neurosci 2017;39:338-351

DOI: $10.1159 / 000477490$
Kim et al. 


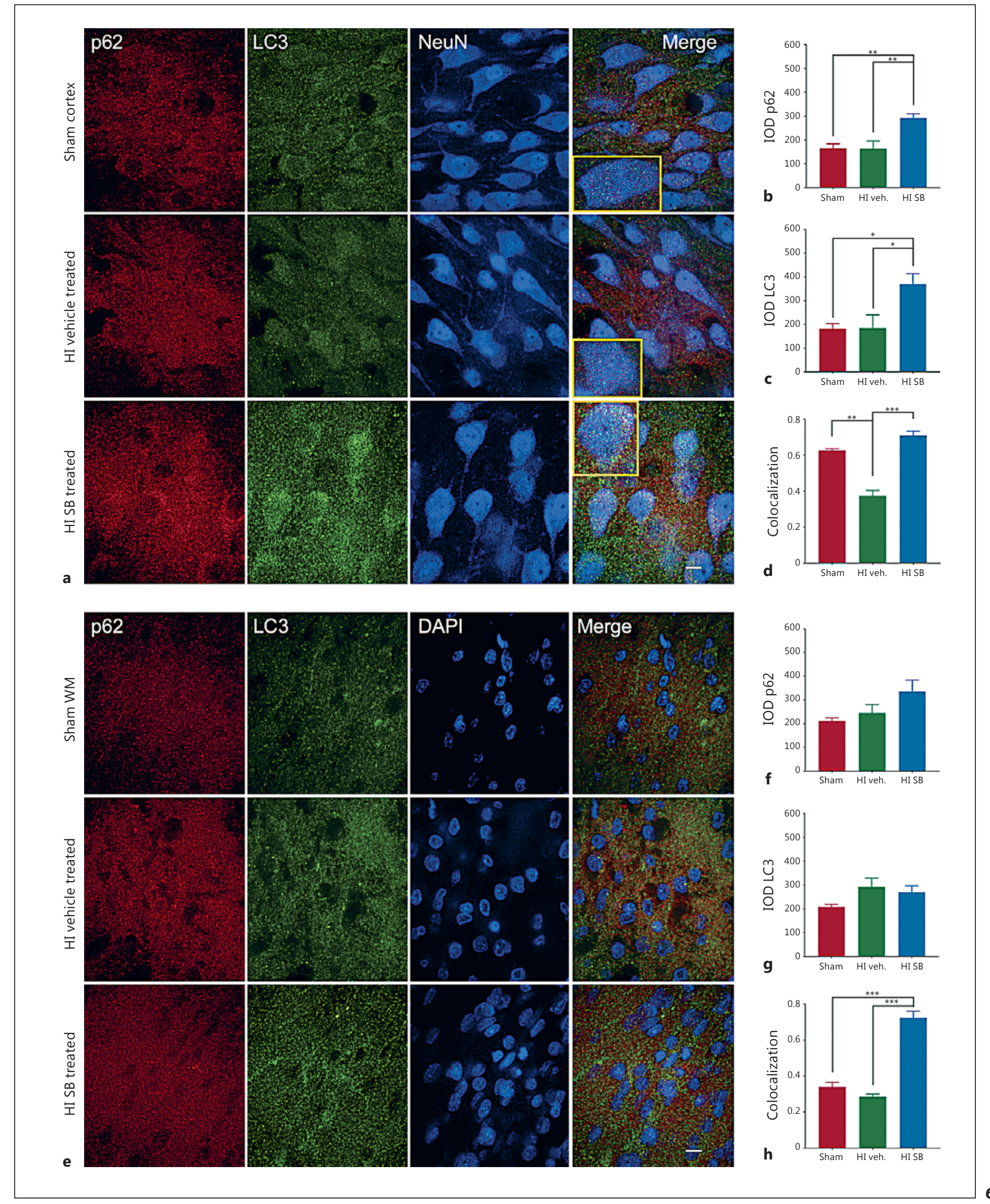






(For legend see next page.) 
efficiently navigate to the hidden platform. The data on path efficiency and total distance traveled eliminates swim speed as a potential confounder for the latency to the platform (Fig. 1b-d); however, the data suggest that the vehicle-treated rats are more hyperactive throughout the 4 training days. Interestingly, children diagnosed with neonatal encephalopathy can present with hyperactivity in addition to poor memory and executive function even in the absence of cerebral palsy motor deficits [25]. Considering SB505124-treated rats had similar swim speeds and travel similar distances as sham rats, it is possible that inhibiting TGF- $\beta$ signaling rescues ventral hippocampal function, associated with anxiety and affect, in addition to preserving the spatial leaning/memory capabilities of the dorsal hippocampus $[26,27]$.

Further tests of anxiety and locomotor hyperactivity will help to elucidate the efficacy of ALK5 inhibition in promoting neuropsychological health. Interestingly, sham animals reached a maximum path efficiency (mean \pm SEM) of $0.234 \pm 0.021$ on training day 4 (a perfectly efficient path would receive a score of 1) suggesting a sizable margin of improvement to reach the platform. This denotes the possibility of other confounders that cannot be explained by the traditional measures used in MWM or may even suggest that rats prefer to explore the pool rather than escape it. Therefore, while we did not deem it necessary to include a group of naïve (healthy control) rats in our studies, our results suggest that including naïve rats may have provided useful baseline measurements on latencies, swim velocity, and path efficiency.

Contrary to our expectations, our data indicate that delayed TGF- $\beta$ type 1 receptor inhibition in the term HI brain injury model exacerbates neurodegenerative processes. In our studies, we started drug administration 3 days after $\mathrm{HI}$ as our earlier studies indicated that this was the time point when TGF- $\beta_{1}$ levels peaked [9]. Contrary to our previous data set, systemic administration of SB505124 to P9 HI-injured rat pups for 7 days did not improve sensorimotor function (Fig. 2) or spatial learning and memory function (Fig. 4). Rather, the SB505124-

Fig. 7. SB505124 stimulates the association of autophagic markers LC3 and p62 following hypoxic-ischemic (HI) injury on P6 in the hippocampus and thalamus. One week after HI injury on P6, samples of the injured forebrain were analyzed by immunofluorescence. SB505124 or vehicle was administered via osmotic pump beginning 3 days after injury and maintained for 4 days prior to intracardiac perfusion; $30-\mu \mathrm{m}$ sections were stained with anti-p62 (red), anti-LC3 (green), and anti-NeuN (blue) markers. a, e Hippocampal and thalamic neurons in the ischemic penumbra of in-

ALK5 Inhibition in Hypoxic-Ischemic

Brain Injury treated rats tended to perform worse on the water maze testing than their vehicle-treated counterparts. Combining SB505124 treatment with therapeutic hypothermia also failed to improve functional recovery (Fig. 2, Fig. 4b). Correspondingly, SB505124-treated P9 HI rat pups had increased hippocampal and thalamic neurodegeneration when compared to vehicle-treated P9 HI pups (Fig. 3). Our studies indicate an overall shift in response to TGF- $\beta$ in this model of developmental brain injury where TGF- $\beta$ signaling is detrimental for the P6 HI pups, but moving towards being neuroprotective in the more mature animal. One explanation is that SB505124 treatment on P9 prolongs the inflammatory insult rather than promoting a reparative state, thus, exacerbating neuronal injury.

The data obtained from the P9 HI rat pups are reminiscent of results from studies in models of adult stroke and Toxoplasma infection that have shown that TGF- $\beta_{1}$ functions as an anti-inflammatory cytokine and that inhibiting TGF- $\beta$ signaling in astrocytes exacerbates lesion size $[28,29]$. Similarly, our data from the P6 HI rat pup model complement data from studies by Nobuta et al. [23], who showed that systemic inflammation induced by lipopolysaccharide injection on $\mathrm{P} 2$ produced a delay in CNS myelination associated with overexpression of TGF- $\beta_{1}$ (produced by activated microglia). They further showed that a single injection of the ALK5 antagonist SB431542 commensurate with lipopolysaccharide injection restored myelin development to approximately $50 \%$ of control. To date, it has not been clear when the effects of TGF- $\beta_{1}$ signaling change, thus our data demonstrating that this switch occurs around P9 in the rat pup provide new and important insights into cytokine signaling in the immature brain. It is possible that a shorter duration of SB505124 treatment in the P9 HI model will improve outcome, which will be important to test in future studies. While our studies do not reveal the molecular changes that are responsible for the different effects of TGF- $\beta$ with age, the most parsimonious explanation is that the astrocyte response to TGF- $\beta$ flips from pro- to anti-inflammatory as the astrocytes mature. As this conversion occurs

jury, respectively. Insets depict representative cells enlarged an additional $\times 2$. b, c, f, $\mathbf{g}$ Integrated optical densities (IOD) of p62 and LC3 fluorescence of sham, vehicle-treated HI, and SB-treated HI groups in each respective region. $\mathbf{d}, \mathbf{h}$ Manders' colocalization coefficient (M1) for the fractional overlap of p62 signals in compartments containing LC 3 signals for the cortex and white matter. $n=$ $3-5$; means $\pm \mathrm{SEM} ;{ }^{*} p<0.05,{ }^{* *} p<0.001,{ }^{* * *} p<0.0001$ (ANOVA followed by the Tukey post hoc test). Scale bars in merged images, $10 \mu \mathrm{m}$.
Dev Neurosci 2017;39:338-351

DOI: $10.1159 / 000477490$
349 
around $\mathrm{P} 9$ in the rat, it likely occurs between gestational weeks 38-40 in the developing human brain.

While we have previously shown that reactive astrogliosis can be attenuated with systemic SB505124 administration, the relationship between this phenomenon and the reduced progression of neuronal death with SB505124 treatment was unknown $[9,11]$. Here, we show that enhanced autophagosome formation may contribute to the reduced degree of secondary neurodegeneration seen with TGF- $\beta$ type 1 receptor inhibition. Given the time line for drug treatment, neuronal LC3/p62 colocalization was still upregulated in SB505124-treated rats 7 days after injury, indicating ALK5 inhibition extended the period of active autophagy past the 5-day mark typical for this type of brain injury in rats $[18,19]$. Autophagy, a method of clearing intracellular debris via lysosomal degradation or sequestration of cytotoxic cargo, is a cytoprotective mechanism for homeostasis and cell survival [30]. Activating autophagy has been shown to promote neuronal survival in vitro and defects in autophagic mechanisms are associated with an inability to eliminate abnormal protein aggregates seen in neurodegenerative conditions such as Alzheimer, Parkinson and Huntington diseases [31, 32]. Therefore, stimulating autophagic sequestration of potentially toxic cellular content and its clearance following cellular stress is a plausible therapeutic strategy to reduce the level of injury.

Currently, there is insufficient evidence to conclude whether activating autophagic pathways following $\mathrm{HI}$ injury is neuroprotective or neurotoxic. Inhibiting autophagy pharmacologically has been shown to reduce acute neuronal cell death in models of perinatal HI [33]. Knocking down Atg7 (a component of the autophagy machinery) in pyramidal neurons of the hippocampus of C57BL/6 mice also improved cell survival following perinatal HI [34], and an analysis of postmortem human brain tissue of asphyxiated infants showed a 7-fold increase in LC3 puncta in dying neurons of basal ganglia compared to noninjured controls, leading to the conclusion that this sharp upregulation of autophagosome assembly may have contributed to neuronal death [35]. However, in these HI studies, severe injuries were evaluated, whereas we evaluated a mild/moderate injury. Thus, one means of reconciling our data with these other data is that autophagy appears to participate in acute neuronal cell death after HI, especially after a severe HI injury through caspase 3-dependent apoptosis. By contrast, after a mild/moderate injury, enhancing autophagy appears to promote cell survival, especially during the secondary or tertiary phase by reducing cell stress and removing damaged organelles.
We suggest that SB505124 treatment may be enhancing autophosome formation by intervening in at least 3 interrelated, anti-autophagic pathways. Firstly, studies performed in the Hewett Laboratories have shown that TGF- $\beta_{1}$ increases astrocytic inducible nitric oxide (NO) synthase production and that antagonizing ALK5 using SB431542 inhibited NO production [24, 36]. NO S-nitrosylates JNK1, a positive modulator of autophagosome production. By inhibiting JNK1 activity, NO prevents core components of the autophagosome from assembling. Initiation of autophagy is dynamically managed by the mechanistic target of rapamycin complex 1 (mTOR) [37]. NO may additionally alter intracellular energy-sensing pathways like mTORC1, the activity of which is induced, to decrease autophagic induction. To wit, Lechpammer et al. [38] showed that downstream mTORC1 targets such as p70/S6 kinase and 40S ribosomal protein S6 exert anti-autophagic effects via STAT3 [39]. Thirdly, we distinguished astrocytes as highly responsive to ALK5 signaling by proliferating and contributing to the proinflammatory phenotype. When ALK5 is antagonized, total numbers of reactive astrocytes are reduced, altogether reducing levels of NO and IL-6 $[9,11]$.

The colocalization of both LC3 and p62 markers is consistent with active autophagosome formation. However, an increase in LC3-II levels is not necessarily indicative of increased active flux as LC3-II levels can increase if autophagosome turnover is disrupted [40]. Therefore, studies involving the use of a lysosomal inhibitor (i.e., chloroquine or pepstatin) must be conducted in future analyses to assess if autophagic flux is affected. In addition, the pathways to injury related to TGF- $\beta$ signaling are most certainly not limited to autophagy. Studies conducted by the Kaufer Laboratory have shown that albumin accumulation in the brain parenchyma secondary to blood-brain barrier dysfunction can lead to aberrant excitatory synaptogenesis/excitotoxicity mediated by astrocytic ALK5 signaling [41], providing yet another avenue of TGF- $\beta$ involvement in delayed cell death [38].

\section{Acknowledgments}

This work was supported by grants from the National Institutes of Health HD052064 and the Leducq Foundation awarded to S.W.L. and by The International Alzheimer's Association (NIRG305325) and the New Jersey Commission on Brain Injury (CBIR14PIL001) awarded to R.D. The authors would like to thank Ms. Clare Ongera for assisting with the MWM experiments.
350

Dev Neurosci 2017;39:338-351

DOI: $10.1159 / 000477490$
Kim et al. 


\section{References}

1 Hagberg H, Mallard C, Ferriero DM, Vannucci SJ, Levison SW, Vexler ZS, et al: The role of inflammation in perinatal brain injury. Nat Rev Neurol 2015;11:192-208.

2 Shankaran S, Laptook AR, Tyson JE, Ehrenkranz RA, Bann CM, Das A, Higgins RD, et al: Evolution of encephalopathy during whole body hypothermia for neonatal hypoxic-ischemic encephalopathy. J Pediatr 2012;160:567572.e3.

3 Gluckman PD, Wyatt JS, Azzopardi D, Ballard R, Edwards AD, Ferriero DM, et al: Selective head cooling with mild systemic hypothermia after neonatal encephalopathy: multicentre randomised trial. Lancet 2005;365:663-670.

4 Shankaran S, Pappas A, McDonald SA, Vohr BR, Hintz SR, Yolton K, et al: Childhood outcomes after hypothermia for neonatal encephalopathy. N Engl J Med 2012;366:2085-2092.

5 Osredkar D, Sabir H, Falck M, Wood T, Maes E, Flatebø T, et al: Hypothermia does not reverse cellular responses caused by lipopolysaccharide in neonatal hypoxic-ischaemic brain injury. Dev Neurosci 2015;37:390-397.

6 Shankaran S: Therapeutic hypothermia for neonatal encephalopathy. Curr Treat Options Neurol 2012;14:608-619.

7 Nishizaki N, Maiguma A, Obinata K, Okazaki $\mathrm{T}$, Shimizu T: Localized intestinal perforations as a potential complication of brain hypothermic therapy for perinatal asphyxia. J Matern Fetal Neonatal Med 2016;29:2537-2539.

8 Polderman KH, Herold I: Therapeutic hypothermia and controlled normothermia in the intensive care unit: practical considerations, side effects, and cooling methods. Crit Care Med 2009;37:1101-1120.

9 Bain JM, Ziegler A, Yang Z, Levison SW, Sen E: TGF $\beta 1$ stimulates the over-production of white matter astrocytes from precursors of the "brain marrow" in a rodent model of neonatal encephalopathy. PLoS One 2010;5:e9567.

10 Buckwalter MS, Wyss-Coray T: Modelling neuroinflammatory phenotypes in vivo. J Neuroinflammation 2004;1:10.

11 Guardia Clausi M, Levison SW: Delayed ALK5 inhibition improves functional recovery in neonatal brain injury. J Cereb Blood Flow Metab 2017;37:787-800.

12 Bona E, Hagberg H, Løberg EM, Bågenholm R, Thoresen M: Protective effects of moderate hypothermia after neonatal hypoxia-ischemia: short- and long-term outcome. Pediatr Res 1998;43:738-745.

13 Patel SD, Pierce L, Ciardiello A, Hutton A, Paskewitz S, Aronowitz E, Voss HU, Moore H, Vannucci SJ: Therapeutic hypothermia and hypoxia-ischemia in the term-equivalent neonatal rat: characterization of a translational preclinical model. Pediatr Res 2015;78:264271.

14 Hamilton N: Quantification and its applications in fluorescent microscopy imaging. Traffic 2009;10:951-961.

15 Dunn KW, Kamocka MM, McDonald JH: A practical guide to evaluating colocalization in biological microscopy. Am J Physiol Cell Physiol 2011;300:C723-C742.

16 Alles YC, Greggio S, Alles RM, Azevedo PN, Xavier LL, DaCosta JC: A novel preclinical rodent model of collagenase-induced germinal matrix/intraventricular hemorrhage. Brain Res 2010;1356:130-138.

17 de Paula S, Vitola AS, Greggio S, de Paula D, Mello PB, Lubianca JM, Xavier LL, Fiori HH, Dacosta JC: Hemispheric brain injury and behavioral deficits induced by severe neonatal hypoxia-ischemia in rats are not attenuated by intravenous administration of human umbilical cord blood cells. Pediatr Res 2009;65:631-635.

18 Carloni S, Buonocore G, Balduini W: Protective role of autophagy in neonatal hypoxiaischemia induced brain injury. Neurobiol Dis 2008;32:329-339.

19 Balduini W, Carloni S, Buonocore G: Autophagy in hypoxia-ischemia induced brain injury. J Matern Fetal Neonatal Med 2012;25(suppl 1):30-34.

20 Klionsky DJ: Coming soon to a journal near you - the updated guidelines for the use and interpretation of assays for monitoring autophagy. Autophagy 2014;10:1691.

21 McKinstry RC, Miller JH, Snyder AZ, Mathur A, Schefft GL, Almli CR, Shimony JS, Shiran SI, Neil JJ: A prospective, longitudinal diffusion tensor imaging study of brain injury in newborns. Neurology 2002;59:824-833.

22 Geddes R, Vannucci RC, Vannucci SJ: Delayed cerebral atrophy following moderate hypoxiaischemia in the immature rat. Dev Neurosci 2001;23:180-185.

23 Nobuta H, Ghiani CA, Paez PM, Spreuer V, Dong H, Korsak RA, Manukyan A, Li J, Vinters HV, Huang EJ, Rowitch DH, Sofroniew MV, Campagnoni AT, de Vellis J, Waschek JA: STAT3-mediated astrogliosis protects myelin development in neonatal brain injury. Ann Neurol 2012;72:750-765.

24 Hamby ME, Coppola G, Ao Y, Geschwind DH, Khakh BS, Sofroniew MV: Inflammatory mediators alter the astrocyte transcriptome and calcium signaling elicited by multiple G-protein-coupled receptors. J Neurosci 2012;32: 14489-14510.

25 van Handel M, Swaab H, de Vries LS, Jongmans MJ: Long-term cognitive and behavioral consequences of neonatal encephalopathy following perinatal asphyxia: a review. Eur J Pediatr 2007;166:645-654.

26 Moser MB, Moser EI: Functional differentiation in the hippocampus. Hippocampus 1998; 8:608-619.

27 Fanselow MS, Dong HW: Are the dorsal and ventral hippocampus functionally distinct structures? Neuron 2010;65:7.

28 Cekanaviciute E, Fathali N, Doyle KP, Williams AM, Han J, Buckwalter MS: Astrocytic transforming growth factor-beta signaling reduces subacute neuroinflammation after stroke in mice. Glia 2014;62:1227-1240.

29 Cekanaviciute E, Dietrich HK, Axtell RC, Williams AM, Egusquiza R, Wai KM, Koshy AA,
Buckwalter MS: Astrocytic TGF-beta signaling limits inflammation and reduces neuronal damage during central nervous system Toxoplasma infection. J Immunol 2014;193:139149.

30 Mizushima N, Komatsu M: Autophagy: renovation of cells and tissues. Cell 2011;147:728741.

31 Benavides GA, Liang Q, Dodson M, DarleyUsmar V, Zhang J: Inhibition of autophagy and glycolysis by nitric oxide during hypoxiareoxygenation impairs cellular bioenergetics and promotes cell death in primary neurons. Free Radic Biol Med 2013;65:1215-1228.

32 Reddy K, Cusack CL, Nnah IC, Khayati K, Saqcena C, Huynh TB, Noggle SA, Ballabio A, Dobrowolski R: Dysregulation of nutrient sensing and CLEARance in presenilin deficiency. Cell Rep 2016;14:2166-2179.

33 Li Q, Li H, Roughton K, Wang X, Kroemer G, Blomgren K, Zhu C: Lithium reduces apoptosis and autophagy after neonatal hypoxia-ischemia. Cell Death Dis 2010;1:e56.

34 Koike M, Shibata M, Tadakoshi M, Gotoh K, Komatsu M, Waguri S, Kawahara N, Kuida K, Nagata S, Kominami E, Tanaka K, Uchiyama Y: Inhibition of autophagy prevents hippocampal pyramidal neuron death after hypoxicischemic injury. Am J Pathol 2008;172:454469.

35 Xie C, Ginet V, Sun Y, Koike M, Zhou K, Li T, Li H, Li Q, Wang X, Uchiyama Y, Truttmann AC, Kroemer G, Puyal J, Blomgren K, Zhu C: Neuroprotection by selective neuronal deletion of Atg7 in neonatal brain injury. Autophagy 2016;12:410-423.

36 Hamby ME, Coskun V, Sun YE: Transcriptional regulation of neuronal differentiation: the epigenetic layer of complexity. Biochim Biophys Acta 2008;1779:432-437.

37 Dunlop EA, Tee AR: mTOR and autophagy: a dynamic relationship governed by nutrients and energy. Semin Cell Dev Biol 2014;36:121129.

38 Lechpammer M, Tran YP, Wintermark P, Martínez-Cerdeño V, Krishnan VV, Ahmed W, Berman RF, Jensen FE, Nudler E, Zagzag D: Upregulation of cystathione $\beta$-synthase and $\mathrm{p} 70 \mathrm{~S} 6 \mathrm{~K} / \mathrm{S} 6$ in neonatal hypoxic ischemic brain injury. Brain Pathol 2016, Epub ahead of print.

39 Qin B, Zhou Z, He J, Yan C, Ding S: IL-6 inhibits starvation-induced autophagy via the STAT3/Bcl-2 signaling pathway. Sci Rep 2015; 5:15701.

40 Barth S, Glick D, Macleod KF: Autophagy: assays and artifacts. J Pathol 2010;221:117-124.

41 Weissberg I, Wood L, Kamintsky L, Vazquez O, Milikovsky DZ, Alexander A, Oppenheim $\mathrm{H}$, Ardizzone C, Becker A, Frigerio F, Vezzani A, Buckwalter MS, Huguenard JR, Friedman A, Kaufer D: Albumin induces excitatory synaptogenesis through astrocytic TGF- $\beta$ /ALK5 signaling in a model of acquired epilepsy following blood-brain barrier dysfunction. Neurobiol Dis 2015;78:115-125.
ALK5 Inhibition in Hypoxic-Ischemic Brain Injury
Dev Neurosci 2017;39:338-351 DOI: $10.1159 / 000477490$ 